\title{
Tratamientos avanzados para la potabilización de aguas residuales*
}

\author{
Angie Tatiana Ortega Ramírez ${ }^{\mathrm{a}}$ - Natalia Sánchez Rodríguez
}

Resumen: el objetivo principal de este artículo es identificar los avances más importantes en referencia a la potabilización de aguas residuales. Para ello, se realiza la explicación detallada sobre la importancia del agua y su estado actual teniendo en cuenta los contaminantes nocivos para el ecosistema acuático. El estudio toma como referentes artículos científicos, revistas, tesis y libros de información desde el año 1936 hasta el 2019 de diferentes bases de datos como Google Académico, Virtual Pro, Ambientalex.info y Science Direct. A partir de la investigación, se describen las ventajas y desventajas que brindan cada uno los tratamientos de agua existentes. Específicamente, se da cuenta de los tratamientos avanzados, en los cuales se determinan las técnicas innovadoras, los beneficios, las aplicaciones y el funcionamiento a escala industrial.

Los resultados de este estudio señalan que al implementar tratamientos avanzados de aguas residuales se genera un desarrollo tecnológico con enfoque en la gestión hídrica, que da lugar a un mayor grado de remoción de contaminantes en el agua. Además, se evidencia que las técnicas de intercambio y tecnologías de membrana son funcionales para realizar procesos de remoción efectivos y eficientes. A pesar de que el método de oxidación avanzada realiza la degradación de contaminantes, se concluye que no es funcional por su alto costo operativo.

Palabras clave: aguas residuales; contaminantes emergentes; intercambio iónico; membranas; oxidación avanzada; tratamientos avanzados

Recibido: $12 / 10 / 2020$

Aceptado: 19/07/2021

Disponible en línea: 31/12/2021.

Cómo citar: A. T. Ortega Ramírez y N. Sánchez Rodríguez, «Tratamientos avanzados para la potabilización de aguas residuales», Cien.Ing.Neogranadina, vol. 31, n. 2 2, pp. 121-134, dic. 2021.

* Artículo de revisión.

a Ingeniera química, ingeniera de Petróleos, especialista en Gerencia de Proyectos, magíster en gestión ambiental para la competitividad. Fundación Universidad de América. Bogotá, Colombia. Correo electrónico: angie.ortega@profesores.uamerica.edu.co ORCID: https://orcid.org/0000-0002-6364-8432

b Ingeniera química. Fundación Universidad de América. Bogotá, Colombia. Correo electrónico: natalia.sanchez@estudiantes.uamerica.edu.co ORCID: https://orcid.org/0000-0001-6993-5750 


\section{Advanced Treatments for Wastewater Purification}

Abstract: This article seeks to identify the most crucial breakthroughs in wastewater purification. For this, a detailed explanation is given about the importance of water and its current state, considering harmful pollutants for the aquatic ecosystem. The study takes scientific articles, journals, dissertations, and textbooks published between 1936 and 2019 in different databases such as Google Scholar, Virtual Pro, Ambientalex.info, and Science Direct. Accordingly, it describes the advantages and disadvantages provided by each of the existing water treatments, specifically advanced treatments, determining innovative techniques, benefits, applications, and operations on an industrial scale. The results suggest that implementing advanced wastewater treatments gives rise to technological development focusing on water management, reaching a higher level of water pollutant removal. Besides, the exchange techniques and membrane technologies are functional to carry out effective and efficient removal processes. Even though the advanced oxidation method degrades pollutants, it is not functional due to its high operating cost.

Keywords: Wastewater; emerging pollutants; ion exchange; membranes; advanced oxidation; advanced treatments 


\section{Introducción}

El agua es considerada como un recurso vital para el desarrollo de los ecosistemas y la vida presente en el mundo. No obstante, tan sólo un 0,5\% de ríos, lagos, corrientes, reservas y aguas lluvia está disponible para su uso [1]. En la actualidad, el uso del agua ha incrementado el doble debido al crecimiento poblacional. Que ha aumentado no solo su demanda, sino también la aparición de agentes nocivos en fuentes acuáticas a causa de la contaminación [2]. Estos desechos provienen de aguas de uso doméstico e industrial [3]. Generalmente, los compuestos que conforman un foco de contaminación en aguas superficiales provienen de plantas industriales farmacéuticas [4], productos de uso personal [5], hormonas esteroides, producción de agroquímicos [6] y restos de medicamentos de uso veterinario [7].

La contaminación de los cuerpos del agua se presenta bajo la existencia de vertimientos orgánicos, sustancias químicas y desechos clorados [8]. Con el incremento de la tasa poblacional a escala mundial, se hace más difícil la eliminación de estos contaminantes emergentes con altos grados de toxicidad. Como consecuencia, se da la aparición de sustancias que no tienen algún reconocimiento con respecto al deterioro de la calidad ambiental que provocan. Esto debido a la alteración molecular que poseen, por lo que no son identificables [9].

Los contaminantes emergentes se definen como compuestos químicos diferentes a la composición del agua, pero cuya presencia en términos de concentración no suele ser representativa debido a su alta hidrosolubilidad [10]. Debido a las cantidades significativas que se han hallado de ellos, con el paso de los años, se han convertido en una situación de gran preocupación por el daño ecosistémico que pueden generar [11]. Al encontrarse en grandes proporciones y con un alto grado de solubilidad, estos contaminantes no suelen tener una remoción completa. Habitualmente, se remueve el sobrenadante de las superficies acuáticas [12]. Además, la mayoría de las plantas que realizan el tratamiento de aguas residuales, no suelen contar con la tecnología que permita generar una remoción eficiente [13]; lo que pone en riesgo el ecosistema acuático y la calidad del agua [14].

En ese sentido, se ha demostrado que los residuos provenientes de elementos de uso diario no poseen ningún tipo de control y que ninguno de ellos ha sido removido en un $100 \%$ [15]. Conjuntamente, debido a que son microcontaminantes que el subsuelo no puede absorber [16], conllevan consecuencias severas al entrar en contacto con el medio acuático [17]. A partir de estos contaminantes, aparecen las aguas residuales que sufren una transformación molecular por la magnitud de residuos suspendidos en ellas [18]. Las aguas están compuestas en un $99 \%$ de agua y el $1 \%$ restante posee sólidos disueltos, suspendidos y coloidales, los cuales aumentan el Dво (Demanda Biológica de Oxígeno) que requieren los cuerpos de agua para degradar la materia orgánica presente en ella. Afectando así a los seres vivos que pertenecen al entorno acuático [19].

Actualmente, los tratamientos aplicados para la potabilización del agua implementan un modelo de economía circular hídrica. Puesto que al purificarla pueden volver a implementarse mediante la recuperación de nutrientes, energía, biomoléculas, metales, compuestos orgánicos e inorgánicos provenientes de estas aguas. Adicionalmente, estos suponen una ventaja para la valorización de aguas residuales porque, en este momento, solo se tiene en cuenta en servicios agrícolas [20]. Para ello, se utilizan los siguientes tratamientos:

- Tratamiento primario: también es denominado como primera sedimentación. Allí se remueven compuestos orgánicos flotantes o suspendidos. En este proceso se busca reducir el DBо en un 30 a $40 \%$ y tiene una duración de 1.5 a 3 horas [21].

- Tratamiento secundario: se trata de procesos biológicos basados en la oxidación mediante 3 métodos: biofiltración, aireación y estanques de oxidación. Por lo general, se suele alcanzar un Dво de 10-30 mg/L [22].

- Tratamiento terciario: remueve, principalmente, fosfatos y nitratos a partir del carbón activado y arena, o utilizando tecnologías avanzadas [22]. 


\section{Metodología}

Para el desarrollo de este artículo, se realizó una búsqueda documental empleando bases de datos como Google Académico, Virtual Pro, Ambientalex.info y Science Direct, de las que se obtuvieron artículos científicos, revistas, tesis y libros que brindan información sobre los tratamientos avanzados de aguas residuales. Para la búsqueda de información en la base de datos Scopus, el operador booleano empleado es "residual water and treatments." En el desarrollo de la investigación, se realizó la búsqueda de información desde el año 1936 hasta el 2019 (figura 1).

\section{Select year range to analyze: $1936 \quad \checkmark$ to $2020 \quad \checkmark \quad$ Analyze}

\section{Documents by year}

50

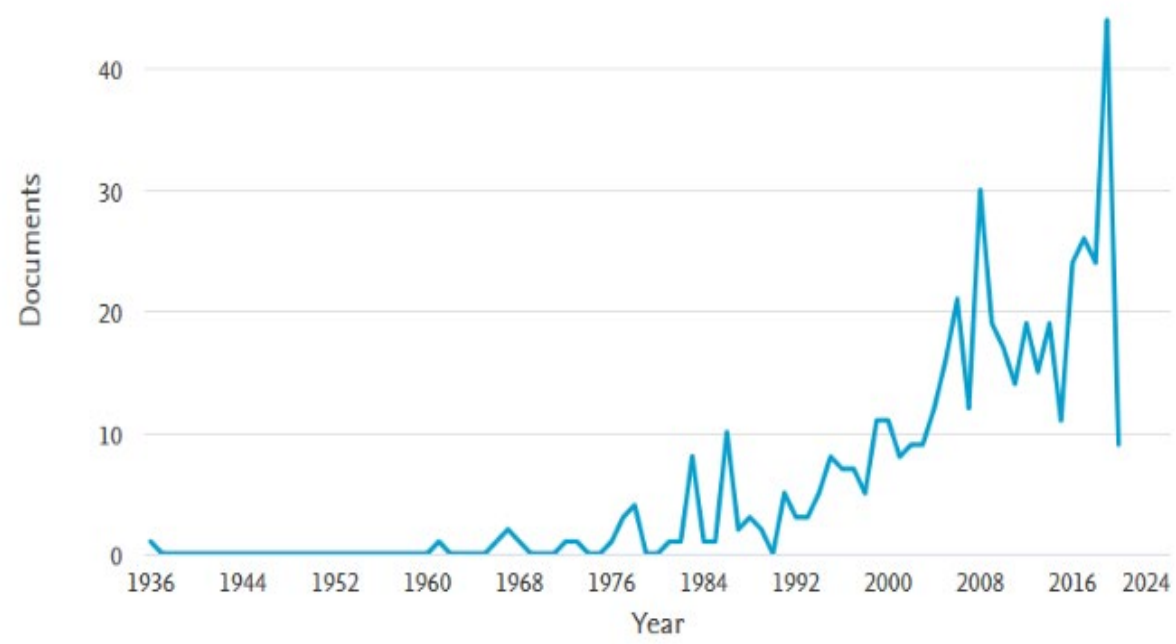

Figura 1. Número de documentos publicados por año.

Fuente: Scopus. Analyze search results. Documentos desde el año 1936.

En la figura 1, se observa que desde el año 1936 inician investigaciones sobre métodos de potabilización. En 2019 se publican 44 artículos, siendo así el período con más información relacionada al tema de interés. Por otra parte, se realiza una búsqueda sobre los autores que más han investigado acerca de los tratamientos de aguas residuales. La figura 2 expone los autores que realizan investigaciones sobre tratamientos de aguas residuales, entre los que destaca el autor Adesogan, A.T, quien ha publicado 4 artículos, de los cuales 3 cuentan con la participación de Salawu. 
Documents by author

Compare the document counts for up to 15 authors.

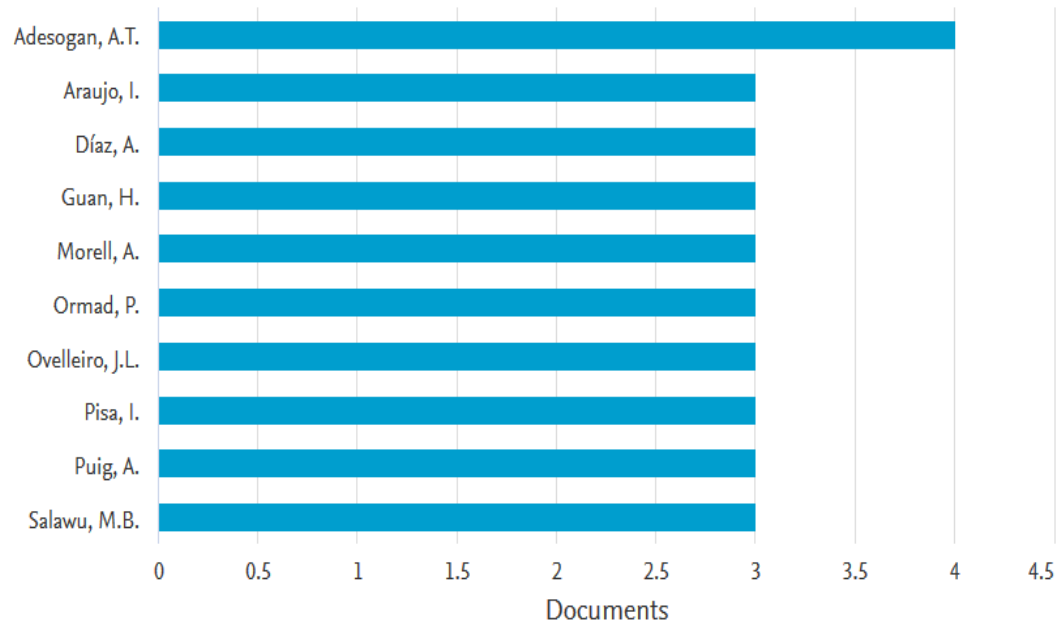

Figura 2. Artículos publicados por el autor.

Fuente: Scopus. Analyze search results. Documents by author.

Una vez realizado esto, se procede a la categorización de la información obtenida en cada base de datos empleada para la investigación (figura 3). En el desarrollo del artículo se utilizaron 63 documentos de los cuales, 42 artículos son pertenecientes a la base de datos de Google académico. De Science Direct, se obtuvieron 10 artículos de investigación experimental. Para el caso de VirtualPro, se emplearon 9 documentos relevantes al tema a tratar. Por último, de la base de datos Scopus solo se empleó un artículo. Cabe mencionar que para la investigación se hizo uso del libro base, procedente de Ambientalex.info. La información recolectada se utilizó con el fin de obtener información representativa sobre investigaciones que se han realizado acerca de la implementación de nuevas tecnologías para la potabilización del agua residual.

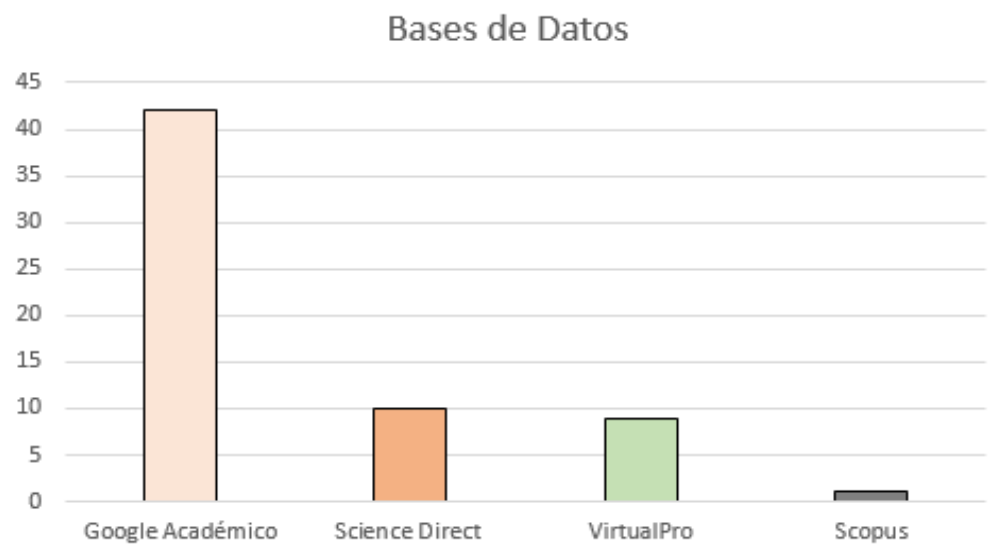

Figura 3. Número de artículos encontrados por cada base de datos.

Fuente: elaboración propia. 
De los 63 artículos reunidos, 37 de ellos están redactados en inglés y los 26 documentos faltantes se encuentran en español. España y México son algunos de los países de los cuales surgen las investigaciones escritas en español. Mientras el mayor número de publicaciones en inglés tiene su origen en Estados Unidos, Alemania y Canadá.

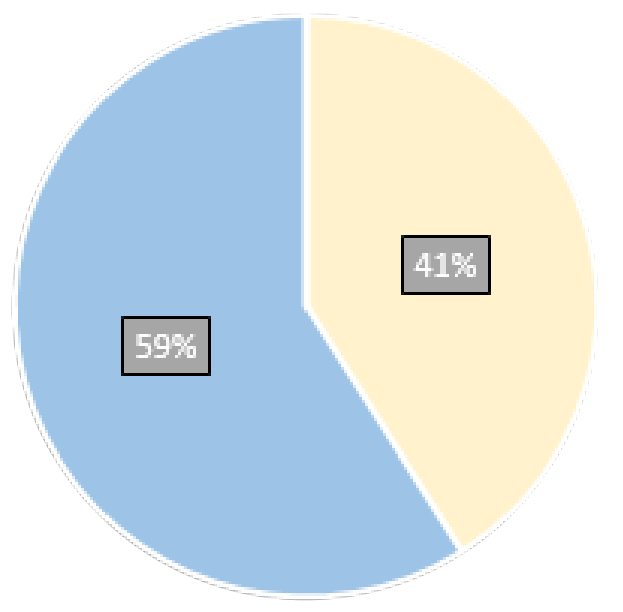

Español

Inglés

Figura 4. Distribución de la información obtenida por idiomas.

Fuente: elaboración propia.

\section{Desarrollo del tema}

Los tratamientos avanzados involucran la implementación de procesos luego del tratamiento secundario en donde se reduce la carga orgánica, presencia de metales y patógenos del agua residual [23]. En cuanto a los aspectos productivos, brindan algunas ventajas como:

- Se adiciona hidróxido de aluminio para disminuir el DBo [23].

- Si hay presencia de metales pesados en el agua, se emplean técnicas de adsorción mediante carbón activado [23].

- Decrece el remanente de fosfato mediante la adición de hidróxido de calcio [23].

- En ocasiones, el agua se solidifica. Por ende, se emplea la aireación para aumentar la solubilidad y proceder a una oxidación de la materia orgánica presente [23].

- En la remoción de los nitratos, se realiza el aumento del pH. Lo que permite que molecularmente se evidencie su separación con el agua [23].

\subsection{Tratamientos avanzados:}

Dentro de los tratamientos avanzados, existen las siguientes técnicas que emplean distintas fuentes tecnológicas para una potabilización adecuada:

\subsubsection{Intercambio Iónico}

El procedimiento se basa en un intercambio de iones disueltos como nitratos, fluoruros o sulfuros y se intercambian por iones de carga similar [24]. Para ello, el agua residual se dispone en un intercambiador que puede ser tanto catiónico como aniónico [25]. El proceso consiste en un intercambio electrostático entre los grupos funcionales del sólido y la resina, lo que conlleva a una desmineralización [24]. Los tipos de intercambiadores existentes se describen en la tabla 1.

Tabla 1. Tipos de intercambiadores iónicos

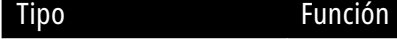

Intercambiadores inorgánicos

\begin{tabular}{ll} 
& $\begin{array}{l}\text { son insolubles en el agua y, } \\
\text { estructuralmente, poseen zeolitas y } \\
\text { arcilla [26]. }\end{array}$ \\
\hline $\begin{array}{l}\text { Intercambiadores } \\
\text { orgánicos }\end{array}$ & $\begin{array}{l}\text { Son redes covalentes de forma } \\
\text { tridimensional que tienen iones } \\
\text { intercambiables asociados con } \\
\text { grupos ácidos o básicos fijos [27]. }\end{array}$
\end{tabular}

Fuente: elaboración propia. 


\section{Resinas de Intercambio Iónico}

Son estructuras poliméricas hechas de poliestireno divinilbenceno que están ubicadas dentro del intercambiador iónico para suavizar o remover minerales de sodio, calcio y magnesio [28]. Los tipos de resina son:

- Resinas catiónicas de ácido fuerte.

- Resinas aniónicas de base fuerte.

- Resinas catiónicas de ácido débil.

- Resinas aniónicas de base débil.

Las resinas están clasificadas como intercambiadores de cationes. Estas tienen un ion movible con carga positiva. Por su parte, las aniónicas poseen una carga negativa [31]. Además, durante su regeneración suelen someterse a diluciones de bases o ácidos fuertes [32].

Tabla 2. Clasificación según su red

\begin{tabular}{ll} 
Tipo & Definición \\
\hline Resinas tipo gel & $\begin{array}{l}\text { Son resinas con micro porosas que bajo } \\
\text { la influencia de un disolvente tienen la } \\
\text { capacidad de dilatarse. Su material está } \\
\text { compuesto por divinilbenceno. Por lo } \\
\text { que tienen una buena resistencia bajo } \\
\text { esfuerzos físicos [29]. }\end{array}$ \\
\hline Resinas & $\begin{array}{l}\text { Tiene un tamaño de poro lo } \\
\text { macroporosas } \\
\text { suficientemente grande para que los } \\
\text { iones entren en contacto con los sitios } \\
\text { activos de la molécula. También están } \\
\text { hechas de divinilbenceno. Pueden ser } \\
\text { oxidativas [30]. }\end{array}$ \\
\hline Resinas isoporosas & $\begin{array}{l}\text { Se caracterizan por su alta } \\
\text { permeabilidad, por lo que les es } \\
\text { facilitado su intercambio. Además, } \\
\text { poseen mayor capacidad que las demás } \\
\text { [30]. }\end{array}$ \\
\hline
\end{tabular}

Fuente: elaboración propia.

\subsubsection{Lecho fijo de intercambio iónico}

Una vez explicado el concepto teórico, se procede a realizar la descripción del montaje de un intercambiador iónico en el cual se lleva a cabo la potabilización del agua residual.

\section{Funcionamiento}

El agua residual que se dirige hacia la zona de alimentación del lecho, es impulsada mediante una bomba centrífuga. Esta va a conectarse con una válvula tipo globo para controlar el caudal de agua que ingresa a la parte superior del intercambiador. El proceso se realiza con el fin de obtener una dispersión de agua uniforme en la resina. Una vez el fluido entra en contacto con la red polimérica (resina), se ejecuta el intercambio de iones que contienen la misma carga. Posterior a ello, el agua se dirige hacia la parte inferior del tanque donde se encuentra una bomba que va a desplazar el agua tratada al 98\% hacia la zona de distribución [33]

\subsubsection{Aplicaciones de los intercambiadores iónicos}

\section{Ablandamiento de aguas}

Se busca disminuir la dureza del agua mediante el intercambio de iones de sodio [34]. Gracias a esto, no se evidencia la aparición de calcio ni de magnesio [35].

\section{Desmineralización de aguas}

Este proceso consiste en la remoción de los sólidos que se encuentran en disolución. Llevando a cabo el intercambio de iones de hidronio y dividiéndolos en ácidos fuertes y débiles. Luego se combina con agua blanda y así brinda la proporción adecuada de sales [36].

\section{Remoción de la alcalinidad}

Generalmente, se lleva a cabo mediante procesos de electrólisis, descomponiendo la molécula de agua en oxígeno y en hidrógeno gaseoso. La alcalinidad suele presentarse por la aparición de iones de calcio y magnesio [37].

\subsubsection{Oxidación Avanzada}

Este método es considerado como un tratamiento de alta competitividad puesto que remueve contaminantes orgánicos como los bio-recalcitrantes e inactivan microorganismos patógenos. Para ello, 
es necesario producir radicales hidroxilos para obtener agentes oxidantes como el $\mathrm{O}_{3}$ (ozono) y el $\mathrm{H}_{2} \mathrm{O}_{2}$ (peróxido de hidrógeno) [38].

Este proceso es funcional debido a que realiza la remoción del contaminante presente. Es decir que no hay una transformación en cuanto a la fase. No hay una generación de residuos y las especies reactantes se descomponen en productos [38]. Dentro de la oxidación se han aplicado varias técnicas para la eliminación de las impurezas. Estas son:

\subsubsection{Incineración}

Técnica catalogada como una oxidación térmica. $\mathrm{Su}$ temperatura de operación se encuentra alrededor de los $800^{\circ} \mathrm{C}$ y posee una capacidad calorífica de aproximadamente $3000 \mathrm{KJ} / \mathrm{kg}$. Dentro de este método, se tienen en cuenta procesos de secado y desgasado, pirólisis y gasificación y, finalmente, la oxidación de los gases que se generan en otras unidades operativas. Para ello, se realiza una combustión empleando hornos de parrilla u hornos rotativos y, así, poder realizar una purificación adecuada [39].

\subsubsection{Oxidación húmeda no catalítica}

Esta técnica realiza la oxidación de efluentes que se encuentran diluidos. El método desempeña la degradación de biosólidos a través de un agente oxidante que le brinda la capacidad de ser un sistema autotérmico. Este mecanismo se realiza mediante cadenas. En donde a partir de una iniciación, se evidencia un ciclo de distribución de oxidación. El proceso de transferencia electrónica se lleva a cabo a una temperatura de $200^{\circ} \mathrm{C}$ y se mantiene una presión de $20 \mathrm{~atm}$. En dicho método, se lleva a cabo una combustión en fase acuosa que no logra la mineralización de la materia orgánica. Lo anterior, genera la producción de especies oxidantes más potenciales. Una vez terminado el proceso, se obtiene un desecho sólido que se somete a un tratamiento ulterior [40].

\subsubsection{Oxidación húmeda catalítica}

Es un método que se utiliza para remover compuestos tanto orgánicos como inorgánicos que se encuentran presentes en el agua. El proceso se basa en emplear reactivos altamente oxidantes y sales. La especie reactiva comúnmente utilizada es el peróxido de hidrógeno y sales de hierro. Gracias a la fusión de estos dos compuestos, se puede llevar a cabo la remoción de efluentes. En caso de no manipular las sales, se realiza la combinación entre el peróxido y el oxígeno, lo que favorece a la reacción puesto que incrementa la velocidad de formación de los grupos hidroxilo [41].

La técnica se caracteriza por tener reacciones rápidas sobre las cadenas carbonadas y permitiendo la formación de radicales orgánicos. Sin embargo, es un método que tiende a ser susceptible ante los cambios bruscos de $\mathrm{pH}$. La condición idónea para ello es que la reacción se encuentre en un medio ácido. Por lo que este valor se debe encontrar en un intervalo de 2,5 a 3,5 , permitiendo que la desintegración del hidrógeno no sea significativa [41].

Para la producción del peróxido y las sales, se pueden utilizar catalizadores tanto homogéneos como heterogéneos. Esto con el fin de mejorar factores operativos como lo son la temperatura, la presión y el tiempo de residencia. Generalmente, se emplea un catalizador sólido ya que incrementa la velocidad de reacción, lo que permite que la remoción de los contaminantes sea eficiente [41]. Comúnmente, el proceso se realiza en la industria de colorantes porque mediante la adición de oxígeno se remueven compuestos nitrogenados que son nocivos para el ecosistema acuático [42].

\subsubsection{Fotocatálisis}

Proceso de degradación del cual se obtiene una reacción fotoquímica que permite la descomposición de la materia orgánica y de los metales pesados que se encuentran en disolución. Para realizarlo se utiliza como fuente de energía la radiación UV proveniente de los rayos solares [43]. Al llevar a cabo el tratamiento, es necesario implementar un catalizador, porque el residuo orgánico presente en el agua no es capaz de captar por sí mismo los fotones que provocan la radiación uv, la cual degrada el contaminante [43]. Dentro de este método, existen dos subdivisiones: 


\section{Fotocatálisis Heterogénea}

El catalizador se encuentra en constante contacto con la luz solar, por lo que permite que se formen los radicales oxidantes para que remueva compuestos tanto orgánicos como inorgánicos. En este, se presentan múltiples reacciones radicales, las cuales surgen a partir de mezclas entre compuestos químicos y mediante la aplicación de una fuente energética. Generalmente, se emplea como especie reactante Dióxido de Titanio, y para el suministro energético se utiliza luz ultravioleta [44].

\section{Fotocatálisis Homogénea}

Es comúnmente denominada como el proceso Fenton, el cual se caracteriza por su alto potencial oxidativo. En este tratamiento la especie reactiva empleada es el oxígeno [45] y es el elemento que efectúa la oxidación produciendo radicales hidroxilos. No obstante, estudios han demostrado que el proceso podría ser más efectivo, empleando peróxido de hidrógeno, hierro u ozono, debido a su potente acción oxidante [46]. Gracias a esto, se remueve el $73 \%$ de los colorantes que se encuentran en el agua, reduciendo el DBO en un 63\% y el DQO en un $53 \%$ [47].

\subsubsection{Ozonización}

Este método se encarga de la remineralización del agua llevando a cabo la eliminación de los compuestos orgánicos mediante la participación del ozono. Inicialmente, se inyecta aire hacia un generador de ozono. La molécula de oxígeno recibe choques eléctricos que buscan disociar la molécula para permitir la formación de ozono. Posterior a ello, el ozono entra en contacto con el agua residual mediante una cámara de contacto. En él, se encuentran dispuestos agitadores para incrementar la velocidad del proceso de degradación de la materia orgánica. Una vez purificada el agua, es necesario destruir el ozono, haciéndolo pasar por una malla para separar las impurezas. Por último, ingresa a un reactor en donde se termina de purificar para que pueda volver a ser recirculado [43]. El tratamiento con ozono es, usualmente, empleado en las industrias farmacéuticas puesto que remueve una gran proporción de antiinflamatorios, antibióticos y estrógenos. Además, es eficaz en el tratamiento de aguas residuales de índole tanto industrial como urbano [48].

\subsubsection{Oxidación electroquímica}

El proceso consiste en la formación de peróxido de hidrógeno como agente oxidante a partir de la inmersión de un ion y un catión de oxígeno en una celda electrolítica, cuyas condiciones operativas se encuentran a una presión de 1 atmósfera y a una temperatura inferior de $100^{\circ} \mathrm{C}$ [43]. El tratamiento se lleva a cabo mediante la generación de cationes que desequilibran las moléculas dispersadas por el choque eléctrico. Haciendo que los ánodos se conviertan en radicales hidroxilos [49]. El método se puede realizar de dos formas (tabla 3 ),

Tabla 3. Tipos de oxidación electroquímica

\begin{tabular}{ll}
\hline $\begin{array}{l}\text { Oxidación } \\
\text { directa }\end{array}$ & $\begin{array}{l}\text { Se utiliza un ánodo que posea una alta } \\
\text { resistencia a la corrosión. La técnica es } \\
\text { aplicable cuando se requiere la degradación de } \\
\text { compuestos orgánicos [43]. }\end{array}$ \\
\cline { 1 - 2 } $\begin{array}{l}\text { Oxidación } \\
\text { indirecta }\end{array}$ & $\begin{array}{l}\text { Hay iones metálicos que operan en un sistema } \\
\text { cerrado con el fin de evitar la contaminación } \\
\text { del agua con partículas externas. Cabe resaltar } \\
\text { que estos iones tienen la habilidad de oxidar } \\
\text { de forma eficiente [43]. }\end{array}$ \\
\hline
\end{tabular}

Fuente: elaboración propia.

\subsubsection{Radiolisis}

En el proceso, los electrones chocan con el agua provocando una pérdida energética para ir desnaturalizando la molécula y así llevar a cabo la separación [43]. Este inicia a partir de la captación de un electrón en un entorno no oxigenado cuya carga es negativa. Luego, se libera un electrón del agua para que haya una interacción entre ellos. Posterior a ello, el electrón que fue liberado, se mantiene en un medio oxigenado [28], que va a interactuar con el oxígeno y dar como resultado un radical $\mathrm{OH}$. De esta manera, se lleva a cabo la potabilización del agua [50]. En esta técnica se busca producir electrones que son sensibles ante una reacción en un medio acuoso. [43]. Esto se efectúa a partir de fotones de una energía muy alta que son provocados por una fuente radioactiva [51]. 


\subsubsection{Irradiación gamma}

La irradiación consiste en la desintegración del material tanto orgánico como inorgánico disuelto en el agua a partir de fuentes que emiten radiación. Usualmente, para dar origen a ello, se emplean elementos como el cobalto y el cesio:

\section{Fuente de cobalto}

Hay una desintegración molecular provocando la aparición de iones de níquel. Luego de ello, las moléculas niqueladas pasan por un proceso de excitación en donde desprenden fotones irradiados, contenidos en rayos gamma [43].

\section{Fuente de cesio}

El elemento sufre una descomposición estructural, la cual deja hallazgos de partículas compuestas de cloruro de cesio que ingresan a un separador. En este, el cesio sale por la zona de fondos de la columna para luego dirigirse a un reactor nuclear que permite llevar a cabo la excitación de la molécula, formando fotones de rayos gamma [43]. Una vez obtenidos los rayos, se ponen en contacto con el agua residual provocando el rompimiento molecular que genera ionizaciones atómicas. Esto permite que las impurezas orgánicas se separen del átomo de agua [52].

\subsubsection{Oxidación utilizando luz ultravioleta}

El proceso consiste en la excitación de electrones a partir de lámparas ultravioleta, cuya longitud de onda debe ser de $190 \mathrm{~nm}$. Al haber realizado dicha excitación, se presenta un fenómeno denominado fotólisis que consiste en la formación de radicales mediante la presencia de átomos de hidrógeno. Lo anterior genera una separación química en donde se visualiza una purificación del agua [43]. Este método consiste en emplear una molécula de peróxido de hidrógeno como agente oxidante. Su objetivo es desintegrarse en dos radicales $\mathrm{OH}$, a partir de una escisión fotolítica provocada por la radiólisis generada para poder llevar a cabo el proceso de remoción de sustancias orgánicas, como lo son los fenoles [53]. Este método es eficiente al momento de retirar cloro residual del agua o para inactivar bacterias, virus y protozoos [54].

\subsubsection{Reactor tubular de oxidación avanzada}

El sistema cuenta con un reactor, que realiza la reacción de oxidación para la purificación de los cuerpos de agua. Dicho reactor cuenta con dos entradas y una salida conectadas a unas válvulas que permiten el paso del flujo. Dentro de este, se encuentra localizada una lámpara ultravioleta [55]. Por otra parte, el montaje tiene una bomba encargada de impulsar las especies reactivas y el agua a purificar.

\section{Funcionamiento:}

El agua residual y el peróxido de hidrógeno ingresan a un mezclador que permite que exista un mayor contacto entre sí. Luego de ello, el fluido ingresa por la parte inferior del reactor en donde entra en contacto con la lámpara UV. Esto provoca la descomposición molecular de la especie oxidante, la cual permite la degradación del material contaminante. Para ello, es necesario emplear sulfato de hierro como catalizador [55].

\subsubsection{Aplicaciones}

Tabla 4. Funciones de las técnicas de potabilización

\begin{tabular}{ll}
\hline Ozonización & $\begin{array}{l}\text { Remoción de contaminantes } \\
\text { emergentes [56]. }\end{array}$ \\
\hline Fenton & $\begin{array}{l}\text { Oxidación en aguas procedentes de } \\
\text { vertimientos [56]. }\end{array}$ \\
\hline $\begin{array}{l}\text { Fotocatálisis y } \\
\text { radiación }\end{array}$ & $\begin{array}{l}\text { Purificación del agua mediante } \\
\text { fotorreactores a partir de la emisión de } \\
\text { ondas radioactivas [56]. }\end{array}$ \\
\hline Todas las técnicas & $\begin{array}{l}\text { Potabilización terciaria del agua luego } \\
\text { de un tratamiento biológico [56]. }\end{array}$ \\
\hline
\end{tabular}

Fuente: elaboración propia.

\subsubsection{Membranas}

Es un método de filtración directa que tiene un gran potencial para dar una buena calidad del 
agua. Permite la recuperación de nutrientes y hacer sostenible el tratamiento. Además, las membranas son selectivas, por lo que resulta ser un proceso eficaz [57]. Las membranas poseen dos clasificaciones; según su composición y según su naturaleza. En cuanto a su composición, las membranas se dividen de la siguiente manera:

Tabla 5. Diversidad en la composición de la membrana

\begin{tabular}{ll}
\hline $\begin{array}{l}\text { Membranas } \\
\text { inorgánicas }\end{array}$ & $\begin{array}{l}\text { Se caracterizan por poseer una buena } \\
\text { estabilidad mecánica, química y térmica. } \\
\text { Dentro de ellas, se encuentran las } \\
\text { membranas metálicas, de vidrio y cerámicas } \\
\text { membranosas [58]. }\end{array}$ \\
\hline $\begin{array}{l}\text { Membranas } \\
\text { orgánicas }\end{array}$ & $\begin{array}{l}\text { Son las más empleadas industrialmente } \\
\text { debido a su gran variedad manera [58]. }\end{array}$ \\
& \\
\hline
\end{tabular}

Fuente: elaboración propia.

En cuanto a su naturaleza, se clasifican así:

Tabla 6. Clasificación de la membrana según su origen

\begin{tabular}{ll}
\hline $\begin{array}{l}\text { Membranas } \\
\text { microporosas }\end{array}$ & $\begin{array}{l}\text { Su tamaño de poro se encuentra en un } \\
\text { rango entre 0,001 y } 10 \mathrm{~mm} \text { [58]. }\end{array}$ \\
\hline $\begin{array}{l}\text { Membranas } \\
\text { eléctricamente } \\
\text { cargadas }\end{array}$ & $\begin{array}{l}\text { Se caracterizan por poseer cargas tanto } \\
\text { positivas como negativas. Estas brindan } \\
\text { el paso de las sustancias con la condición } \\
\text { de que posean la misma carga de la } \\
\text { membrana [58]. }\end{array}$ \\
\hline $\begin{array}{l}\text { Membranas } \\
\text { isótropas }\end{array}$ & $\begin{array}{l}\text { Tienen un espesor menor a 20 mm y } \\
\text { actúan como agentes filtrantes [58]. }\end{array}$ \\
\hline
\end{tabular}

Fuente: elaboración propia.

\subsubsection{Ultrafiltración}

El agua residual pasa a través de las membranas, en donde las partículas sólidas se retienen en las paredes. Sus condiciones operativas, se encuentran bajo presiones de 100-180 KPa y emplean caudales que se encuentran en un rango de 2.5 hasta $25 \mathrm{~m}^{3} /$ día [59]. Luego del tratamiento, las membranas son lavadas con agua caliente. Después, son sometidas en una solución ácido-base y, por último, se les adiciona detergente caliente para regenerarlas [59].

\subsubsection{Osmosis inversa}

En el proceso, el agua residual atraviesa la membrana en donde su posición cambia desde la zona más concentrada hasta la más diluida. En ese transcurso, se va a presenciar una presión diferencial. Por ende, el agua va a tener un movimiento inverso. Logrando así que los contaminantes emergentes queden en la zona diluida y el agua se encuentre potabilizada [60].

\subsubsection{Microfiltración}

Hay una filtración que se realiza mediante un tamizado que se encarga de separar los sólidos suspendidos presentes en el agua. Este se ejecuta por diferencias de diámetro de partícula [61]. La presión operativa se encuentra en un rango de 0,5 a 3 bar y el diámetro de la partícula se estima en un intervalo de 0,05 a 10 micrómetros [61].

\subsubsection{Reactor MBR}

El agua se desplaza a través de fangos activados en donde se evidencia la separación de las partículas sólidas. Este reactor puede operar con altas concentraciones de sólidos suspendidos [62]. Además, cuenta con dos unidades de purificación. Una parte se basa en una purificación biológica para remover la materia orgánica y, la segunda, realiza una filtración para eliminar los sólidos presentes [63].

\subsubsection{Aplicaciones}

- Es empleado para purificar el agua residual caliente.

- Es utilizado para llevar a cabo la regeneración de las proteínas.

- Es partícipe en la separación de aceites que se encuentran en disolución.

\section{Conclusiones}

Los tratamientos terciarios podrían ser implementados en poblaciones de bajos recursos, en donde se vería aplicada la economía circular hídrica a partir del concepto de recirculación del agua y recuperación de nutrientes. Ya que, esto trae desarrollo desde el punto de vista de la triple cuenta, 
sometiéndola a tratamientos no convencionales que permiten su potabilización en vertimientos, tanto de origen industrial como doméstico. No obstante, estos presentan como desventaja el alto costo operativo que acarrean. Además de que, al ser regiones de escasos recursos, es necesario que el gobierno brinde el apoyo financiero para que puedan ponerse en marcha y brindar la calidad de agua adecuada.

El saneamiento del agua residual mediante tratamientos avanzados es eficiente. Sin embargo, en técnicas de purificación por membrana es recomendado realizar una segunda filtración. Asegurando que no queden residuos de la misma. En el caso de la oxidación avanzada, es necesario realizar muestreos que aseguren la ausencia de peróxidos en el agua tratada y en la técnica de intercambio iónico. En ese sentido, no se evidencia ningún tratamiento posterior puesto que se realiza una potabilización completa.

\section{Referencias}

[1] B. Baker, A. Omer y C. Aldrige, "Water: availability and use" en Water Conservation Education, Oxford (Mississippi): ResearchGate 2016, pp. 1-5.

[2] Z. Kilic, "The Importance of Water and Conscious Use of Water", International Journal of Hydrology, vol. 4, $\mathrm{n}^{\circ}$ 5, pp. 1- 3, 2020.

[3] Z. Hasan y S. Jhung, "Removal of Hazardous Organics from Water Using Metal-Organic Frameworks (MOFs): Plausible Mechanisms for Selective Adsorptions", Journal of Hazardous Materials, vol. 283, n. ${ }^{\circ}$ 1, pp. 329-339, 2015, febr. 11.

[4] N. Khan, S. Ullah, S. Ahmed et al., "Recent Trends in Disposal and Treatment Technologies of Emerging-Pollutants: A Critical Review", Trac Trends in Analytical Chemistry, vol. 122, n. ${ }^{\circ}$ 1, pp. 115 - 744, 2020, en.

[5] N. Tran, M. Reinhard y K. Gin, "Occurrence and Fate of Emerging Contaminants in Municipal Wastewater Treatment Plants from Different Geographical Regions: A Review" Water Research, vol. 133, n. ${ }^{\circ} 1$, pp. 182-207, 2018, abr. 15.

[6] H. Quesada, A. Baptista, L. Cusioli et al., "Surface Water Pollution by Pharmaceuticals and an Alternative of Removal by Low-Cost Adsorbents: A Review", Chemosphere, vol. 222, n. ${ }^{\circ} 1$, pp. 766-780, 2019, my.
[7] L. Charaud, E. Jarde, A. Jaffrezic et al., "Veterinary Pharmaceutical Residues from Natural Water to Tap Water: Sales, Occurrence and Fate", Journal of Hazarouds Materials, vol. 361, n. ${ }^{\circ}$ 1, pp. 169-186, 2019, en. 5.

[8] J. Vigo, Efecto de microorganismos eficaces (ME) en el proceso de depuración de aguas residuales domésticas en condiciones altoandinas, Lima: Universidad Peruana Union, 2020.

[9] J. Sánchez, J. Velasco, L. Belmonte et al., "The Worldwide Research Trends on Water Ecosystem Services", Ecological Indicators, vol. 99, n. ${ }^{\circ}$ 1, pp. 310-323, 2019, abr.

[10] L. Nascimiento, R. Araújo y L. Alvarez, “Contaminantes orgánicos emergentes: impactos y soluciones para la salud humana y el medio ambiente", Contaminantes emergentes: Impactos y soluciones, vol. 1, n 24, pp. 2834, 2015.

[11] M. Arguello, J. Mendoza, A. Tintos et al., Ecotoxicological Analysis of Emerging Contaminants from Wastewater Discharges in the Coastal Zone of Cihuatlán, Jalisco, Mexico, Jalisco, México: Water, 2019.

[12] J. Sousa, A. Ribeiro, M. Barbosa et al., "A Review on Environmental Monitoring of Water Organic Pollutants Identified by Eu Guidelines", Journal of Hazardous Materials, vol. 344, n. ${ }^{\circ}$ 1, pp. 146-162, 2018, febr. 15.

[13] P. Carmona, Estudio de la presencia, ocurrencia y destino final, de un compuesto farmacéutico (amoxicilina) en una planta de tratamiento de agua residual institucional educativa, Bucaramanga, Colombia: Universidad Pontificia Bolivariana, 2019.

[14] V. Propp, A. D. Silva, C. Spencer et al., "Organic contaminants of emerging concern in leachate of historic municipal landfills", Environmental Pollution, vol. 276, n. ${ }^{\circ} 1$ pp. 116-474, 2021 my. 1.

[15]T. Rasheed, M. Bilal, F. Nabeel et al., "Environmentally-Related Contaminants of High Concern: Potential Sources and Analytical Modalities for Detection, Quantification, and Treatment", Environmental International, vol. 122, n. ${ }^{\circ} 1$, pp. 52-66, 2019, en.

[16] R. Hendili, A. Alatrache, M. Attia et al., "Antibacterial Inactivation of Spiramycin After Titanium Dioxide Photocatalytic Treatment", Comptes Rendus Chimie, vol. 20, n 7, pp. 710-716, 2017, jul.

[17] S. Rathi, S. Kumar y P. Show, "A Review on Effective Removal of Emerging Contaminants from Aquatic Systems: Current Trends and Scope for Further Research," Journal of Hazardous Materials, vol. 409, n. ${ }^{\circ}$ 1, pp. 124-413, 2021, my. 5.

[18] Ú. Moses, Fiscalización Ambiental vinculada a las aguas residua les, Piura; oEfA, 2016. 
[19] I. Bokova, G. Ryder y S. Uhlenbrook, Aguas residuales. El recurso desaprovechado, París: Unesco, 2017.

[20] S. Guerra, P. Oulego, E. Rodríguez et al., Towards the Implementation of Circular Economy in the Wastewater Sector: Challenges and Opportunities, Madrid: Water, 2020.

[21] S. Oakley, Preliminary Treatment and Primary Sedimentation, Michigan: Unesco, 2018.

[22] Organica, "Primary, secondary and tertiary wastewater treatment: How do they work?", 2017, jun. 27. [Internet]. Disponible en https://www.organicawater.com/primary -secondary-tertiary-wastewater-treatment-work/. [Accedido: 2021, jun. 28].

[23] G. Pepper, "Municipal wastewater treatment" en Environmental and Pollution Science; $3^{\text {a }}$ edición, Amsterdam Boston: Elsevier/Academic Press, 2019, pp. 393-418.

[24] Fluence, "What is ion exchange?" 2021, febr. 16. [Internet]. Disponible en https://www.fluencecorp. com/what-is-ion-exchange/. [Accedido: 2021, jun. 28].

[25]Carbotecnia, Intercambio iónico, Jalisco, México: Centro de aprendizaje, 2020.

[26] C. Cobrazu y V. Inlezakis, "Inorganic ion exchange" en Progress in FIltration and Separation, vol. 21, Loughborough, England: Academic Press, 2015, pp. 425-498.

[27] C Luca, "Organic ion exchangers" en Encyclopedia of separation science, London: Academic Press, 2019, pp. 1617-1632.

[28] J. Patel, Ion Exchange Resins, Mumbai: ResearchGate, 2016.

[29] S. Caján y S. Villegas, Influencia del caudal de un drenaje ácido de mina sobre la remoción de metales pesados utilizando resinas de intercambio iónico, Cajamarca, Colombia: Universidad Privada del Norte, 2020.

[30] E. Villanueva, Determinación de la cinética de adsorción de fructooligosacátidos, a partir del extracto de yacón, empleando resinas de intercambio iónico y carbón activado, Tacna, Perú: Escuela profesional de ingeniería en industrias alimentarias, 2017.

[31] S. Al-Asheh y A. Aidan, A Comprehensive Method of Ion Exchange Resins Regeneration and Its Optimization for water Treatment, Waste Water Treatment, vol. 1, n. ${ }^{\circ} 1$, pp. 1-14, 2020.

[32] R. Sharma, "Handbook on water and waste water treatment" in Ion Exchange. Refreshing the Planet, India: Ion India 2014.
[33] DuPont, Ion Exchange Resins, United States: DUPONT, 2019.

[34] T. Scherer, Water Softening (Ion Exchange), Riga, Latvia: NDSU, 2017.

[35] Watex, Water Softening, watex, Technological solutions and Equipment for Water treatment, 2015.

[36] D. Hermosilla, C. negro y A. Blanco, "Water demineralization" en Encyclopedia de Memnranas, Madrid: Universidad de Madrid, 2015.

[37] J. Bajgai, Simple Method of Increasing the Alkalinity and Reduction Power of Water by Boiling Simple Method of Increasing the Alkalinity and Reduction Power of Water by Boiling, Yonsei: ResearchGate, 2019.

[38] J. Cardenas, B. García, A. Sánchez et al., "Wastewater Treatment by Advanced Oxidation Process and Their Worldwide Research Trends", International Journal of Environmental Research and Public Health, vol. 17, n. o 1, p. 170, 2020.

[39] F. Sanz, Eficiencia energética en plantas depuradoras de aguas residuales urbanas: valorización de lodos por incineración, Cartagena, Colombia: Escuela Técnica Superior de Ingeniería Industrial, 2018.

[40] A. García, Valorización de fangos de depuradora mediante la oxidación húmeda, Gandía, España: Escuela Politécnica Superior de Gandía, 2018.

[41] P. Riaño, Degradación del cristal violeta presente en aguas residuales mediante la oxidación catalitica húmeda con peróxido de hidrógeno (CWPO) a partir de óxidos mixtos de $\mathrm{Mn}$-Cu a condiciones moderadas, Bogotá: Fundación Universidad de América, Facultad de ingenierías, 2018.

[42] S. Álvarez, V. Águeda, J. Delgado et al., Oxidación húmeda catalítica. Proceso medioambientalmente favorable en la eliminación de contaminantes presentes en aguas residuales, Bogotá: Grupo Ingenio Colombiano, 2018.

[43] S. Bes, Manual técnico sobre procesos de oxidación avanzada aplicados al tratamiento de aguas residuales industriales, Tarragona, España: CYTED, 2018.

[44] H. Ortega, Estudio de la eliminación de diclofenaco en aguas residuales mediante fotocatálisis heterogénea con TiO2, Valladolid: Universidad de Valladolid, Departamento de Ingeniería Química y Tecnología del Medio Ambiente, 2017.

[45] A. Aquino, Tratamiento de aguas residuales de la industria textil artesanal por fotocatálisis homogénea y heterogénea, Huancayo, Perú: Universidad Nacional del Centro de Perú, Unidad de Posgrado de la Facultad de Ingeniería Química, 2017. 
[46] A. Vorontsov, "Advancing Fenton and Photo-Fenton Water Treatment Through the Catalyst Design", Journal of Hazardous Materials, vol. 372, n. ${ }^{\circ}$ 1, pp. 103112, 2019, jun. 15.

[47] W. Bae, H. Won, B. Hwang et al., "Characterization of Refractory Matters in Dyeing Wastewater During a Full-Scale Fenton Process Following Pure-Oxygen Activated Sludge Treatment", vol. 287, n. ${ }^{\circ}$ 1, pp. 421428, 2015, abr. 28.

[48] J. Urbina y J. Vera, "Los contaminantes emergentes de las aguas residuales de la industria farmacéutica y su tratamiento por medio de la ozonización”, Informador Técnico, vol. 84, n. ${ }^{\circ}$ 2, pp. 249-263, 2020, febr. 2.

[49] K. Espinosa y K. Maldonado, Aplicación de oxidación electroquímica y ozonización para el tratamiento de aguas residuales de una empacadora de pescado, Guayaquil, Ecuador: Universidad de Guayaquil, Facultad de Ingeniería Química, 2019.

[50] A. Puerta y J. Morales, "Efectos biológicos de las radiaciones ionizantes", Revista Colombiana de Cardiología, vol. 27, n. ${ }^{\circ}$ 1, pp. 61-71, 2020, mzo.

[51] O. García, Rompimiento molecular del fenol en agua residual mediante alta frecuencia de ultrasonido, Zaragoza, España: Benemérita Universidad Autónoma de Puebla, Facultad de ciencias físico matemáticas, 2020.

[52] E. Cruz, Degradación de un bactericida por radiación gamma acoplado a un tratamiento biológico, Cuyo, Argentina: Biblioteca Leo Falico V, 2017.

[53] D. Pelayo, Procesos de oxidación avanzada: avances recientes y tendencias futuras, Cantabria, España: Escuela Técnica Superior de Ingenieros Industriales y de Telecomunicación, 2018.

[54] M. Palacios, Análisis de los vórtices Taylor-Couette en un reactor tubular para la desinfección de aguas mediante luz ultravioleta, Cuernavaca, México: Instituto de investigación en ciencias básicas y aplicadas, 2019.

[55] M. Terán, Estudio de la aplicación de procesos de oxidacion avanzada a aguas contaminadas, Catalunya,
España: Universidad Politécnica de Catalunya, Departamento de Ingeniería Química, 2016.

[56] S. Chiva, J. Berlanga, R. Martínez et al., Procesos de oxidación avanzada en el ciclo integral del agua,Valencia, España: Universitat Jaume I 2017.

[57] S. Hube y B. Wu, Direct membrane filtration for wastewater treatment and resource recovery: A review, in Science of The Total Environment, vol. 710, Iceland: Elsevier, 2020.

[58] L. Palacio y A. Hernández, Determinación de cambios estructurales mediante microscopía de fuerza atómica en superficies tratadas con plasma de gases, Valladolid, España: Universidad de Valladolid, Facultad de Ciencias, 2017.

[59] Solis, Velez y Ramírez, "Membrane Technology: U1trafiltration", Entre Ciencia e Ingeniería, vol. 11, n. ${ }^{\circ} 22$, pp. 26-36, 2017.

[60] S. Jiang y B. Ladewig, "A review of Reverse Osmosis Membrane Fouling and Control Strategies", Science of The Total Environment, vol. 595, n. ${ }^{\circ}$ 1, pp. 567-583, 2017.

[61] M. Suárez, Efecto del tratamiento de microfiltración por membranas en el proceso de elaboración de vino y bebidas alcohólicas a base de vino, Cuenca, España: Universidad del Azuay, Facultad de Ciencia y Tecnología, 2020.

[62] M. Kalaruban, P. Loganathan, W. G. Shim et al., "Mathematical Modelling of Nitrate Removal from Water Using a Submerged Membrane Adsorption Hybrid System with Four Adsorbents", Applied Sciences, vol. 8, n. ${ }^{\circ} 2$, p. 194, 2018, en. 29.

[63] E. Vásquez, Estudio de biorreactor de membrana para el tratamiento de aguas residuales urbanas, Sevilla, España: Escuela Politécnica Superior, 2015. 